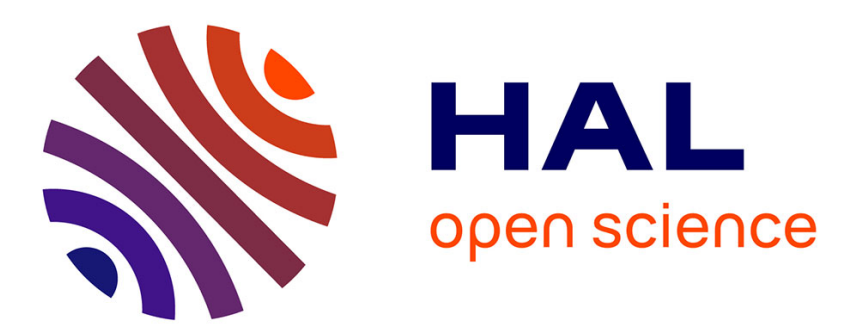

\title{
Damage localization using transmissibility functions: A critical review
}

\author{
Simon Chesné, Arnaud Deraemaeker
}

\section{To cite this version:}

Simon Chesné, Arnaud Deraemaeker. Damage localization using transmissibility functions: A critical review. Mechanical Systems and Signal Processing, 2013, 38 (2), pp.569-584. 10.1016/j.ymssp.2013.01.020 . hal-00807823

\section{HAL Id: hal-00807823 \\ https://hal.science/hal-00807823}

Submitted on 17 Mar 2016

HAL is a multi-disciplinary open access archive for the deposit and dissemination of scientific research documents, whether they are published or not. The documents may come from teaching and research institutions in France or abroad, or from public or private research centers.
L'archive ouverte pluridisciplinaire HAL, est destinée au dépôt et à la diffusion de documents scientifiques de niveau recherche, publiés ou non, émanant des établissements d'enseignement et de recherche français ou étrangers, des laboratoires publics ou privés. 


\title{
Damage localization using transmissibility functions: a critical review
}

\author{
Simon Chesné \\ Université de Lyon, CNRS INSA-Lyon, LaMCoS UMR5259, F-69621, France, simon.chesne@insa-lyon.fr \\ Arnaud Deraemaeker \\ Université Libre de Bruxelles, BATir, 50 av F.D. Roosevelt CP 194/2 B-1050 Brussels, aderaema@ulb.ac.be
}

\begin{abstract}
:
This paper deals with the use of transmissibility functions for damage localization. The first part is dedicated to a critical review of the state-of-the-art highlighting the major difficulties when using transmissibility functions for damage detection and localization. In the second part, an analytical study is presented for non-dispersive systems such as chain-like mass-spring systems. The link between the transmissibility function and the mechanical properties of four subsystems defined by the boundary conditions, the position of the excitation and the two measurement locations used for the computation of the transmissibility functions is derived. This result is used to discuss the situations in which damage localization is likely to work. The last section discusses the extension of these results to more general dispersive systems such as beams or plates.
\end{abstract}

\section{Keywords:}

Structural Health Monitoring, Damage localization, Transmissibility functions 


\section{Introduction}

Many countries are facing serious security problems due to the aging of their civil infrastructures, such as bridges. On one hand, the structures are coming to the end of their theoretical lifetime, and on the other hand, the maximum loads to which they are exposed are always increasing (i.e. traffic on bridges). It is therefore crucial to be able to estimate the current state of health of such structures, as well as their remaining lifetime. Current techniques are based on scheduled maintenances with visual inspections and local methods such as ultrasounds or eddy currents.

For more than twenty years, researchers have developed alternative global methods based on the measurement of vibration signals. Existence of structural damage in an engineering system leads to modification of the vibrations. The main idea is that damage changes the stiffness of the structure and therefore the modal properties (natural frequencies, mode shapes and modal damping values) which can be obtained from results of dynamic (vibration) testing. The goal of structural health monitoring (SHM) is to detect damage at or near its onset, before it becomes critical to structure's function and integrity. One of the major challenges in detecting and locating small-scale structural damage is that this type of damage is a local phenomenon. Rytter [1] distinguished four levels of damage identification each of which provides more detailed information about the structural damage. The ultimate goal of the previous work in SHM is to determine when, where, and how badly a structure is damaged or deteriorated. The literature on the subject is enormous [2] but these methods have not seen a big success, mainly for two reasons: (i) the modal properties of the structure are also dependant on environmental parameters (weather, loads, wind ...) which cause changes in the vibration characteristics of the same order of magnitude as damage, and (ii) mode shapes and frequencies are global properties which are not very sensitive to small localized damages.

Thanks to the enormous advances in sensors and instrumentation, it is now possible to instrument a structure with hundreds or thousands of sensors which are capable of measuring the vibrations of structures in real time. By following the dynamic behavior of the structure in real time, it is possible to apply statistical methods in order to detect small damages and remove the effects of the environment ([3], [4], [5] and [6]). This opens the way to the development of automated damage detection systems and could allow going from an "on schedule" maintenance to an "on demand" maintenance, therefore greatly reducing the costs and the risks of failure between two maintenances.

The next step to help the engineers in the maintenance of structures is to develop tools to locate damage. There exist two families of methods: the first family uses a model (i.e. finite elements) which is updated regularly based on experimental results [7]. Any change in the parameters of the model will give the indication of the position of damage. The major drawback with these methods is the difficulty to build accurate models of the initial structure.

The second family of methods relies only on the experimental measurements to detect damage. In order to do this, it is important to choose the right feature. The ideal feature is one that is defined locally and changes only if damage appears at that specific location. To the best of our knowledge, for vibration based methods, two types of features have been identified and investigated in the past: curvature mode shapes ([8], [9], [10] and [11]) and transmissibility functions. 
This paper deals with transmissibility functions (TF). The first section is dedicated to a critical review of the literature on the use of TFs for damage detection and localization. This section highlights the major issues for the development of SHM systems based on TFs: the importance of the choice of the frequency bands, and the problem of operational and environmental variability which might cause false alarms. In addition, we point out the lack of general analytical results to assess the limits of validity of the damage localization methods based on TFs.

The second section is dedicated to a review of the mathematical concepts of TFs and the associated damage indicators proposed in the literature. The third section deals with a specific type of systems: chain-like massspring systems. A general analytical result is derived in which the TF is expressed as a function of the properties of four sub-systems defined by the locations of the measurement points used for the TF, and the location of the force. This analytical formula is used to understand the impact on the TFs of a change of stiffness or mass parameters in each of the four sub-systems and to draw conclusions on the limits of the methods proposed for damage localization. The formula can be extended to more general non-dispersive systems such as rods in traction-compression or in torsion.

The last section is dedicated to more general types of systems for which the simple analytical formula obtained for chain-like mass spring systems cannot be derived. A beam example is presented in order to emphasize the fact that the applicability of the method is, in this case, even more restricted than for the simple non-dispersive systems studied in the previous section. It seems difficult however to derive more precisely these additional restrictions.

\section{Review of transmissibility-based damage detection and localization methods}

Transmissibility functions have been first proposed as potential features for damage detection in [12]. Since then, they have been extensively used in the research group lead by Keith Worden at the University of Sheffield for damage detection and localization. In [13], an auto-associative neural network using TFs was developed to compute a novelty index for damage detection. The example treated was a numerical 3 degrees of freedom (dofs) chainlike mass spring system. Another numerical example involving a 3 dofs system was studied a few years later by the same research group [14]. In this contribution, outlier analysis based on frequency lines in the TFs was used for damage detection. A few years later, experimental validations on the use of TFs for damage detection ([15], [16] and [17]) and localization [18] have been performed using vibration data from a laboratory wing box structure and a gnat aircraft wing. Detection was based on three different novelty detection techniques (outlier analysis, auto-associative neural networks and kernel density estimation), while localization was based on supervised learning using a multi-layer perception (MLP) neural network. Apart from the fact that TFs appear to have a high sensitivity to damage, the main motivation for using them is the fact that there is no need to measure the excitation. Although this is true, it should be emphasized that for the types of structures encountered in aeronautics and civil engineering applications, TFs do not depend on the frequency content of the excitation, but do depend on the location of the excitations. A change in the excitation location might cause a significant change in the TFs under normal (undamaged) condition, which is very likely to cause false alarms. This issue was recently discussed in [19] where it was shown that TFs computed at the eigenfrequencies of the system are independent on the excitation location. Note 
that this property has also been used to develop output-only modal analysis techniques [20]. Restricting the TFs to narrow frequency bands around these eigenfrequencies was therefore proposed as a way to get rid of the variability of the TFs due to a change in the location of the excitation.

In the studies cited above, the authors have used specific frequency lines or bands extracted from the TFs. For damage detection, one should find the frequency bands for which the features are highly sensitive to damage and insensitive to variability in the normal condition [15]-[16]. For damage localization, an additional requirement is to find frequency bands in which the features are highly sensitive to one type of damage, and almost insensitive to the others [18]. A major problem is that these frequency bands cannot be determined $a$ priori without having access to data from the structure in the different damage conditions and that in practice, such data is rarely available. This problem has been recognized by the research group at the University of Sheffield who has proposed the use of pseudo-faults in the form of added masses on the actual structures in order to produce data representative of the structure in the damaged condition [21]-[22]. Although this approach has proved to work on specific examples, there is no evidence of its applicability on any given type of structure.

While all these studies show, on specific examples, the possibility to use TFs for damage detection and localization, they do not present any analytical results supporting these findings. While it is rather simple to demonstrate the sensitivity of TFs to damage (because they are the ratio of FRFs which are known to be sensitive to damage), it is much more difficult to prove the localization capabilities of TFs.

Around the same time, similar developments on the use of TFs (called transmittance functions in the related papers) for structural health monitoring have been presented in [23], [24], [25], [26] and [27]. A damage indicator based on the integral over a frequency band of the difference between the intact and the damaged TFs was used for damage detection and localization. The underlying (heuristic) idea is that damage between points $i$ and $j$ will cause a change in the TF computed between these two points, and not (or only slightly) on the other TFs. The applicability of the technique was assessed using experimental data acquired on beams, plates and wind turbine blades. Although the method was shown to be efficient for the examples treated, there are again no analytical developments to assess the limits of applicability of the technique. In particular, the issue of the specific frequency bands to be used for successful damage localization is not raised.

In parallel to these investigations, several authors have published analytical work on the extension of the transmissibility concept, initially developed in the field of vibration isolation for two degrees of freedom systems, to multi-degree of freedom systems ([28], [29] and [30]). In particular, in [29], the authors have demonstrated the fact that when generalizing the concept of transmissibility to MDOF systems, the transmissibility matrix depends on the location of the excitation, as stated above. In addition, it can easily be shown that the resonances and antiresonances of TFs correspond to the zeros of the frequency response functions (FRFs) of the two sensors from which the transmissibility is computed. A study of the sensitivity of these zeros to stiffness changes can be found in [31]. This study does not show a strong difference in sensitivity between the poles and zeros of FRFs, at least on the numerical example treated, which somehow contradicts the statements of other authors (see for example [32]). This contradiction could show that the issue is very problem dependent so that care should 
be taken when generalizing a result from a few examples only.

Based on these theoretical developments, the possibility to detect and locate damage using TFs has been studied by the research team of Maia at IST in Porto [33]. The difference of TFs between the undamaged and the damaged conditions was used for damage localization, in a very similar way to the method presented in [25]. One important aspect of these methods is the fact that they are based on the data measured on the intact structure only, therefore avoiding the need of training data emphasized in [14], [15] and [16]. The authors note however that while the approach seems to work well at low frequencies, the results deteriorate when increasing the frequency band, raising again the question of the frequency band to be used. In practice, the authors found that one should look at the difference between the TFs only below the first resonance or antiresonance, which restricts strongly the usable frequency range. An alternative proposed is to 'count the occurrences' instead of computing the cumulative error in a frequency band. Very recently in [34], an alternative damage indicator based on correlations of the TFs rather than differences, similar to the well known modal assurance criterion (MAC) used in modal analysis, has been proposed for damage detection (but not for localization). The results show a higher sensitivity when TFs are used compared to FRFs. In all these examples, while the method seems to be effective on the numerical example studied, it is difficult to assess the general applicability of the approach.

In parallel to the work of the research group of Maia, the use of TFs for damage localization has also been studied by the group of Adams at Purdue University [32], based on the analytical developments in [29][31] and the method proposed earlier in [23], [24] and [25]. In addition to dealing with linear systems, the authors extend the application of TFs to non-linear systems. For linear systems, the possibility to locate damage is shown on a 3 dofs chain-like mass-spring system, for which it is demonstrated that damage between masses $i$ and $j$ causes a change in the transmissibility between these two masses only. Note however that there are restrictions on the position of the excitation for this result to be valid. While this is probably the only tentative to derive analytical expressions to demonstrate the localization properties of TFs, the authors claim that this result can be generalized to systems with more degrees of freedom, but there is no proof presented. Further papers by the same research team deal with applications on a steel frame structure, a helicopter frame [35], and rolling tires [36]. A later paper [37] deals with the issue of frequency band selection in order to reject variability due to operational and environmental conditions, emphasizing one more time this important aspect. Here again, data acquired on the damaged structure is necessary in order to select the frequency bands.

Although the present paper is intended to deal with linear structures only, it is worth citing a recent study on the extension of the concept of transmissibilities for non-linear systems using the concept of "non-linear output frequency response functions" (NORF) [38].

\section{General formulation}

This first part introduces the basic equations used in this work. It details the formulation of frequency response functions, necessary to understand the concept of TFs and of the damage indicator.

\subsection{Frequency response functions}

The usual $\mathrm{n}$ dof undamped system, illustrated in Figure 1, is written for the case of harmonic excitation as: 


$$
\left(\mathbf{K}-\omega^{2} \mathbf{M}\right) x=f
$$

Where $\mathbf{K}$ and $\mathbf{M}$ are the stiffness and mass [nxn] matrices and $\mathbf{f}$ and $\mathbf{x}$ are the input and output (response) [nx1] vectors. The nature of the model of the system will determine the structures of matrices $\mathbf{K}$ and M. Next sections will show that particular properties of TFs depend on these structures. The displacement is given by

$$
x=\frac{\operatorname{adj}\left(\mathbf{K}-\omega^{2} \mathbf{M}\right)}{\operatorname{det}\left(\mathbf{K}-\omega^{2} \mathbf{M}\right)} f=\mathbf{H} f
$$

Where $\mathbf{H}$ is the dynamic flexibility matrix, and the $j, k^{\text {th }}$ term of the adjoint matrix is

$$
(-1)^{k+j} \operatorname{det}\left(\left[\mathbf{K}-\omega^{2} \mathbf{M}\right]_{j k}\right)
$$

The subscript $j k$ on the matrix $\left[\mathbf{K}-\omega^{2} \mathbf{M}\right]_{j k}$ denotes the fact that the $k^{\text {th }}$ column and the $j^{\text {th }}$ row have been deleted. Note that the subscript $k$ is linked to the input as illustrated in Figure 1.

It is known that the poles are the natural frequencies of the system, and represent a global behaviour of the structure; the poles are the eigenvalues of $\left(\mathbf{K}-\omega^{2} \mathbf{M}\right)$. Poles appear when the denominator $\operatorname{det}(\mathbf{K}$ $\left.\omega^{2} \mathbf{M}\right)$ is zero.

Zeros on frequency response function for a force at coordinate $k$ and response at coordinate $j$ occur when the respective adjoint element (element at column $k$ and row $j$ ) is zero due to a singularity of the submatrix $\left[\mathbf{K}-\omega^{2} \mathbf{M}\right]_{\mathrm{jk}}$. The zeros are the eigenvalues of the submatrix $\left[\mathbf{K}-\omega^{2} \mathbf{M}\right]_{\mathrm{jk}}$, which are potentially different for each inputoutput pair.

\subsection{Transmissibility functions}

On the contrary to admittance functions (usually called frequency response functions FRF) which are frequency responses between conjugate variables (motion response/ force input), TFs are obtained by taking the ratio of two response spectra of like variables (motion response/motion input) $x_{i}$ and $x_{j}$, for a given input located at degree of freedom (DOF) $k$ :

$T_{i j}^{k}(\omega)=\frac{x_{i}^{k}(\omega)}{x_{j}^{k}(\omega)}=\frac{(-1)^{k+i} \operatorname{det}\left(\left[\mathbf{K}-\omega^{2} \mathbf{M}\right]_{i k}\right)}{(-1)^{k+j} \operatorname{det}\left(\left[\mathbf{K}-\omega^{2} \mathbf{M}\right]_{j k}\right)}$

It can be observed that the common denominator $\operatorname{det}\left(\boldsymbol{K}-\omega^{2} \boldsymbol{M}\right)$, whose roots are the system's poles, disappears by taking the ratio of the two response spectra. Consequently, poles or zeros of the TF correspond to the zeros of both FRFs.

In general, TFs depend on the input location of the (unknown) force. Note that this definition corresponds to the case of a single point input, which may not cover all cases encountered in practice. It is however the most widely used in SHM applications. Moreover, the limitations of this approach which are highlighted in the present study hold for multiple-input locations, which have been found to be an even more restrictive case. For the sake of clarity, this study is therefore restricted to the single input location case.

\subsection{Damage indicator}

Damage detection and localization is based on the tracking of the changes of the TFs at certain frequencies or over certain frequency bands. For that purpose, a damage indicator was introduced first in [23] and used by many authors subsequently. This damage indicator $D_{i j}$ is a scalar value which quantifies the change of the TF between measurement points $i$ and $j$ across a given frequency band. It is defined over a frequency range from $\omega_{1}$ to $\omega_{2}$ by: 


$$
D_{i j}\left(\omega_{1}, \omega_{2}\right)=\frac{\int_{\omega_{1}}^{\omega_{2}}\left|T_{i j}^{h}(\omega)-T_{i j}^{d}(\omega)\right| d \omega}{\int_{\omega_{1}}^{\omega_{2}}\left|T_{i j}^{h}(\omega)\right| d \omega}
$$

where $T^{h}{ }_{i j}$ is the baseline undamaged TF, and $T^{d}{ }_{i j}$ is the current potentially damaged TF. As stated earlier, the selection of an appropriate frequency range $\left[\omega_{1}, \omega_{2}\right]$ is a key parameter for a good detection, as discussed in different papers in the literature. Note that a slightly different version of this indicator has been proposed in [35] where the amplitude of the logarithm of the TFs is used instead of the TFs. In practice TF based on accelerations measurement are often used. Due to the definition of the TF, their expression is identical using accelerations or displacements, since the multiplying factor $\omega^{2}$ cancels out.

\section{Non dispersive systems}

\subsection{Definition}

The first class of problem that we shall consider is governed by a second order partial differential equation. This kind of problem presents non dispersive waves, including transverse vibrations of strings, longitudinal deformation of beams, or chainlike mass-spring systems. The system is governed by the well known wave equation. For simplicity, in the following, discussions on non dispersive systems will be illustrated with chain-like mass-spring systems.

\section{$3.2 \quad N$ degree of freedom system}

The chain-like mass-spring systems are models composed of masses interconnected by springs, capable of only translational motion. Each node has only one degree of freedom and is connected to its nearest neighbours. Such a simple model allows for a good understanding of various properties of TFs for non dispersive systems.
A $n$ degree of freedom chain-like mass-spring is represented in Figure 1.

These systems are well known, and their equation of motion has the form of:

$\mathbf{H}^{-1} x=\mathbf{Z} x=f$

With $\mathbf{Z}$ is the following 3 diagonal [nxn] matrix:

$\mathbf{Z}=\left[\begin{array}{ccccc}M_{1}(\omega) & -k_{2} & & & \\ & \ddots & & & \\ & -k_{j} & M_{j}(\omega) & -k_{j+1} & \\ & & & \ddots & \\ & & & -k_{n} & M_{n}(\omega)\end{array}\right]$

And where

$M_{i}(\omega)=k_{i}+k_{i+1}-\omega^{2} m_{i}$

Using Eq (2), the FRF between output $j$ and input $k$ can be written:

$H_{j k}=\frac{x_{j}}{f_{k}}=\frac{(-1)^{k+j} \operatorname{det}\left([\mathbf{Z}]_{j k}\right)}{\operatorname{det}(\mathbf{Z})}$

It is interesting here to develop the adjoint matrix (we recall here that it corresponds to the matrix $\mathbf{Z}$ without the $k^{\text {th }}$ column and the $j^{\text {th }}$ row, illustrated with the doted lines):

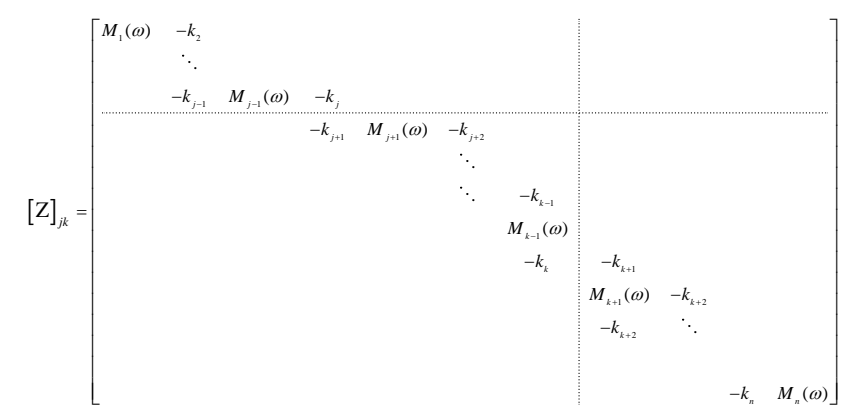

We can write this matrix in the following form:

$[\mathbf{Z}]_{j k}=\left[\begin{array}{cc:c}M a_{j} & M d & \\ \hdashline & M b_{j k} & M e \\ & & M c_{k}\end{array}\right]$ 
Where the three sub matrices of interest, separated by the row $j$ and the column $k$, are:

$$
\begin{aligned}
M a_{j} & =\left[\begin{array}{ccc}
M_{1}(\omega) & -k_{2} & \\
& \ddots & \\
& -k_{j-1} & M_{j-1}(\omega)
\end{array}\right] \\
M b_{j k} & =\left[\begin{array}{ccc}
-k_{j+1} & M_{j+1}(\omega) & \\
& \ddots & M_{k-1}(\omega) \\
0 & & -k_{k}
\end{array}\right] \\
M c_{k} & =\left[\begin{array}{ccc}
M_{k+1}(\omega) & -k_{k+2} & \\
& \ddots & \\
& -k_{n} & M_{n}(\omega)
\end{array}\right]
\end{aligned}
$$

It can be observed that matrices $M d$ and $M e$ are empty, expect at one corner where a single stiffness term appears. Nevertheless their determinant is zero so that they do not appear in the expression of the FRF

The concept of subsystem appears clearly in equation (11). Noting that the sub matrix $M b_{j k}$ is triangular, it follows that the FRF $\mathrm{H}_{\mathrm{jk}}$ can be written as:

$$
H_{j k}=\frac{x_{j}}{f_{k}}=\frac{\operatorname{det}\left(M a_{j}\right) \operatorname{det}\left(M b_{j k}\right) \operatorname{det}\left(M c_{k}\right)}{\operatorname{det}(\mathbf{Z})}=\frac{\operatorname{det}\left(M a_{j}\right) \operatorname{det}\left(M c_{k}\right)}{\operatorname{det}(\mathbf{Z})} \prod_{p=j+1}^{k}\left(-k_{p}\right)
$$

It is interesting to notice that the zeros of the FRF $\mathrm{H}_{\mathrm{jk}}$, do not depend on the dynamic parameters of the system between points $j$ and $k$. The zeros correspond to the poles of the exterior subsystems when dofs $j$ and $k$ are grounded as shown by (12) and (13). Figure 2 illustrates the two exterior subsystems which determine the zeros of the FRF $H_{j k}$.

With these results and observations, we can write the TF for this type of mechanical systems:

$$
T_{i j}^{k}(\omega)=\frac{H_{i k}(\omega)}{H_{j k}(\omega)}=\frac{\operatorname{det}\left(M a_{i}\right)}{\operatorname{det}\left(M a_{j}\right)} \prod_{p=i+1}^{j}\left(-k_{p}\right)
$$

The expression of $T_{i j}{ }^{k}(\omega)$ in equation (14) is not a function of the excitation coordinate $k$, which means that $T_{i j}{ }^{k}(\omega)$ is not dependent on the excitation position as long as we have $k \geq j \geq i$. This independence on the force location is true as long as the force remains on the same side of points $i$ and $j$.

\subsection{Damage localization using TFs}

If the poles are the natural frequencies of the systems, what is the physical meaning of the zeros? The information provided by the zeros seems to be a bonus because it refers to a different system (sometimes called a fictitious system) which can be represented by the original model matrix representation when certain rows and columns have been eliminated (through the adjoint matrix of the numerator). Miu [39] considers that zeros of the FRF represent the resonant frequencies associated with energy storage characteristic of this subportion of the system defined by artificial constraints imposed by the location of input and output. They represent the frequencies at which energy is trapped in the energy storage elements of the subportion of the original system such that no output can ever be detected at the measurement point.

In the context of damage detection, we saw that the system (global) transfer function poles are sensitive to changes in structural health anywhere because the term $\operatorname{det}(\boldsymbol{Z})$ in (9) is a function of all the system parameters. In contrast, the zeros are only sensitive to a certain subset of mass and stiffness parameters that are localized in specific regions of the structure (see equation (14)). This difference in sensitivity to global/local changes can be exploited by selecting a damage feature based on TFs, which are independent of the poles and solely dependent on zeros. By focusing on the zeros rather than the poles, it might be possible to "trap" damage or local structure variation between certain DOFs and perform damage localization. Let us illustrate this with the subsystems (A, B, C and D) represented on Figure 3. The global system is limited by two fixed boundaries, and 3 particular DOFs, (1 input and 2 outputs) delimiting 4 subsystems. 
We consider that outputs are not on the boundary or collocated to the input.

Applying equation (14), we find:

$$
T_{i j}(\omega)=\frac{\operatorname{det}(A)}{\operatorname{det}(A B)} \prod_{p=i+1}^{j}\left(-k_{p}\right)
$$

This expression is of great interest, as it allows understanding the physical origin of poles, zeros and gains of the TF. The first thing to note is that the TF is not dependent on subsystems $\mathbf{C}$ or $\mathbf{D}$ and will not be affected by a damage located in these subsystems.

According to (15) a change of stiffness in subsystems $\mathbf{A}$ or $\mathbf{B}$ will affect $T_{i j}(\omega)$. The possibility to locate damage depends on the sensitivity of $T_{i j}(\omega)$ to stiffness changes in $\mathbf{B}$ due to two sources:

- A change in the gain of $T_{i j}(\omega)$ through the term $\prod_{p=i+1}^{j}\left(-k_{p}\right)$ which is the product of the spring stiffnesses between points $i$ and $j$.

- A change in the poles of $T_{i j}(\omega)$ through the term $\operatorname{det}(\mathbf{A B})$.

On the other hand, a damage in subsystem $\mathbf{A}$ will also cause a change in $T_{i j}(\omega)$ which will be detrimental to the localization. The changes are due to two sources:

- A change in the zeros of $T_{i j}(\omega)$ through the term $\operatorname{det}(\mathbf{A})$.

- A change in the poles of $T_{i j}(\omega)$ through the term $\operatorname{det}(\mathbf{A B})$.

We can conclude from this, that if damage is present anywhere between the position of the applied force and the boundary condition, perfect localization is never possible, unless the damaged spring is located directly next to the applied force. In this very specific case, whatever the values of $i$ and $j$, the damage is never in subsystem A. In all other cases, damage will cause a change in the poles and zeros of $T_{i j}(\omega)$ for some values of $i$ and $j$ (i.e. when $i$ and $j$ are at the right of the damaged spring). In some cases, this leads to the impossibility to locate damage, as will be demonstrated in the examples. Figure 4 summarizes the role of each subsystem.

\subsection{Analysis of a damage detection case}

In this section, a 4 dofs mass-spring system is considered (Figure 5, $\mathrm{m}_{1,2,3}=10^{-3}$ $\left.\mathrm{kg}, \mathrm{k}_{1,2,3}=10 \mathrm{Nm}^{-1}\right)$.

Two representative damage cases are presented. The first case (Figure 6 and Figure 7) where the stiffness $k_{3}$ is damaged (30\%) is the ideal case for damage detection where the input is next to the damaged spring. The damage indicator $D_{i j}$ is computed using (5) and using a large frequency range $[0$; 300 $\mathrm{rad}_{\mathrm{r} . \mathrm{s}^{-1}}$ containing all poles and zeros of the TFs. The interpretation of the results is based on the subsystem representation previously proposed. Figure 8 is an illustration of how the subsystems are rearranged for $\mathrm{T}_{34}$.

$\mathrm{T}_{12}$ and $\mathrm{T}_{34}$ are unchanged, as shown by their null damage indicator. Indeed, for these TFs, the damage is located in a subsystem which has no effect on the TF (C or D). Equation (15) shows that TFs are not sensitive to a structural variation located in these subsystems. $T_{23}$ contains the damaged stiffness. In this configuration, damage is located in subsystem B and the TF is affected by its presence, as shown by the non-zero damage indicator. This case is considered as the ideal one because the damage indicator is always zero except for the TF which contains the damaged element between its outputs.

The second illustrative case is more problematic. The damage is on stiffness $\mathrm{k}_{2}$. The various TFs and damage indicators are shown in Figure 9 and Figure 10.

The interesting result of this case is the fact that the damage indicator of $\mathrm{T}_{23}$ is not zero, although it is smaller than the damage indicator of $T_{12}$. This result 
illustrates the dependence of the $\mathrm{TF}$ on a subsystem outside the domain delimited by its outputs as predicted previously.

In conclusion, for chain-like massspring systems, the TF between points $i$ and $j$ is very sensitive to changes in subsystem $\mathbf{B}$, delimited by these two points. Unfortunately, it is also sensitive to changes in subsystem $\mathbf{A}$, located between point $i$ and the nearest boundary. Consequently, for good damage localization, a particular attention has to be paid on this domain. The numerical results on the simple system presented show that despite this problem, the damage indicator is maximal in the damaged region, so that localization is possible.

\subsubsection{Effect of the frequency range}

One another key parameter to consider is the frequency range used for the computation of the damage indicator. In order to observe its behavior, let's plot its evolution as a function of $\omega$ for a 7 DOF system. This new damage indicator is then defined as:

$$
D_{i j}(\omega)=\frac{\int_{0}^{\omega}\left|T_{i j}^{h}\left(\omega^{\prime}\right)-T_{i j}^{d}\left(\omega^{\prime}\right)\right| d \omega^{\prime}}{\int_{0}^{\omega}\left|T_{i j}^{h}\left(\omega^{\prime}\right)\right| d \omega^{\prime}}
$$

The input force is localized on mass 6. For the first simulation, damage is localized on $\mathrm{k}_{6}$ directly next the applied force. Figure 11shows the evolution of the 6 damage indicators as a function of $\omega$. This corresponds to the special case where the only $\mathrm{TF}$ which changes due to the damage is $T_{56}(\omega)$. The damage only affects the poles and the gain of $T_{56}(\omega)$ since only subsystem $\mathbf{B}$ is damaged, and not subsystem $\mathbf{A}$. Note the sharp increase of $T_{56}(\omega)$ around $50 \mathrm{rad} / \mathrm{s}$ due to the presence of the first pole in this TF.
This first pole corresponds to the first eigenfrequency of the subsystem consisting of 5 masses.

For convenience, in the following, the damage indicators referring to a domain containing the fault are plotted using dotted lines.

In Figure 12, we plot the evolution of the 6 damage indicators as a function of $\omega$ when damage is located on $\mathrm{k}_{3}$. In this case, all damage indicators between point 2 and point 6 are affected. Localization is only possible below $50 \mathrm{rad} / \mathrm{s}$ where $T_{23}(\omega)$ is larger than all the other indicators. As stated earlier, around $50 \mathrm{rad} / \mathrm{s}$ which corresponds to the eigenfrequency of the subsystem consisting of the first five masses, $T_{56}(\omega)$ increases sharply. A sharp increase in $T_{23}(\omega)$ occurs at a higher frequency, around $80 \mathrm{rad} / \mathrm{s}$ corresponding to the first eigenfrequency of the subsystem consisting of the first two masses. For this reason, between 50 and 80 $\mathrm{rad} / \mathrm{s}$, localization is not possible. This type of frequency behavior was also observed in [33] in which the authors have noted that the results of damage localization tended to deteriorate when increasing the frequency band. They also noted that one should stay below the first resonance or anti-resonance of the TF which is coherent with the results presented above.

In general, if the position of the force is not known, it is safe to consider the first eigenfrequency of the whole system as an upper limit for the computation of the damage indicator. In the example considered, this first eigenfrequency is equal to 39.01 $\mathrm{rad} / \mathrm{s}$.

Figure 13 and Figure 14 show the damage indicators, for two different frequency ranges. In Figure 13, the damage indicator uses the whole frequency range $[0,300] \mathrm{rad} / \mathrm{s}$. Localization is not possible. Figure 14 shows that by restricting the frequency band to $[0,39] \mathrm{rad} / \mathrm{s}$ (below the first eigenfrequency of the system), 
localization can be achieved, although as discussed earlier, the damage indicators corresponding to undamaged zones are not equal to zero.

\subsubsection{Effect of added masses on transmissibility functions}

From equation (15), it is clear that a change of mass will have a different effect on $T_{i j}(\omega)$ than a stiffness change. The main difference is that there will not be any change in the gain due to the stiffness terms. The mass change will however modify the poles and zeros of $T_{i j}(\omega)$. We saw in the previous section that good localization was possible at low frequencies thanks to the change in the gain of $T_{i j}(\omega)$ so that we should expect to lose the localization capability for a mass change. This is an important issue, as it is quite common in practical experimental validation exercises, to replace the damage by added masses in order to avoid actually damaging the test structure (see for example [22]). The primary justification for such a procedure is that an added mass will shift the eigenfrequencies down, in a similar way that a stiffness reduction will do. The developments below show that this 'fake damage' technique should not be used to test a damage localization method using transmissibilities, especially if one uses the TFs at low frequencies.

Let's consider the system presented in Figure 15 . The system is constructed with 13 masses and the displacements on masses $1,3,5 . ., 13$ are measured. It is then possible to introduce a mass change between two measurement points. In the next simulations an increase of $30 \%$ of mass 4 is introduced.

Figure 16 shows the evolution of the six damage indicators for an increase of mass $(+30 \%)$ on mass 4 , corresponding to the case of a modification between measurement points 3 and 5 for the 13 dofs system. One sees clearly that localization is not possible at low frequencies. At high frequencies (Figure 17), localization seems improved, but it is never as good as for a stiffness change when considering the damage indicator at low frequencies. The first eigenfrequency of the system is $22 \mathrm{rad} . \mathrm{s}^{-1}$. In order to compare this kind of damage with previous section, Figure 18 shows the damage indicators for $\omega \in[0,21] \mathrm{rad} . \mathrm{s}^{-1}$. It clearly appears that a mass variation cannot be localized.

Of course, localization could still be possible if one restricts the frequencies of interest for the computation of the damage indicator to some specific frequencies at which the damage indicator over the damaged region is much higher than in the other regions. Let us consider the 'single frequency' damage indicator $D s f$ given by :

$D s f_{i j}(\omega)=\frac{\left|T_{i j}^{h}(\omega)-T_{i j}^{d}(\omega)\right|}{\left|T_{i j}^{h}(\omega)\right|}$

For the same example with 13 masses, Figure 19 and Figure 20 show the value of $\operatorname{Dsf}(\omega)$ when respectively the mass addition is located on $m_{4}$ and the stiffness reduction is on $k_{4}$, both between measurement points 3 and 5. It can be seen from the figure that localization is possible only in very limited frequency bands. This emphasizes again the importance of the choice of frequency bands for damage localization, and the fact that these frequency bands cannot be determined a priori without any knowledge on the damaged state. It can also be seen that there is no evidence that a mass change has a somehow similar effect on $\operatorname{Dsf}(\omega)$ than a stiffness change.

\subsection{Discussion}

We have studied the use of TFs for damage localization in chain-like mass spring systems. Based on a simple formula relating the change of TFs to the damage in the massspring system, we have shown that perfect localization of damage is only possible when 
the damage is located directly next to the point of application of the force. In all other cases, damage localization is not perfect and tends to deteriorate when the frequency band used for the computation of the damage indicator is increased. We have shown numerically that for these other cases and frequencies lower than the first eigenfrequency of the system, the damage indicator computed between points $i$ and $j$ is maximum when damage is located between points $i$ and $j$. In this case, damage localization seems to be possible, although there is no guarantee. For higher frequencies, localization is much more problematic and much care should be taken in the selection of the frequency bands of interest for the computation of the damage indicator. Unfortunately, it does not seem possible to determine these frequency bands without $a$ priori knowledge on the response of the damaged structure.

Based again on the simple formula derived, we have compared the effect of a stiffness with the effect of a mass change on TFs for chain-like mass-spring systems. The results show that a mass change has a very different impact on the TFs so that localization is not guaranteed, even at low frequencies. It is therefore advised not to use 'fake damage' in experimental setups by using added masses when using TFs.

Extreme caution should therefore be taken in the use of TFs for damage localization. Even for very simple chain-like mass-spring systems, perfect localization is guaranteed only in very specific conditions (the damage is exactly next to the applied force), and good localization seems to be feasible (but not certain) only by looking at $\mathrm{T}_{\mathrm{ij}}{ }_{\mathrm{ij}}(\omega)$ at frequencies below the first natural frequency of the system, providing that the structural modification is a stiffness change, and not a mass change.

\section{$4 \quad$ Dispersive systems}

\subsection{System characteristics}

In previous sections the theory of the use of TFs to localize damage has been detailed for non dispersive systems. Such systems are characterized by the fact that only one transfer path is available. Unfortunately, this characteristic is rarely present in real life structures. Without this assumption the independence of the TFs with respect to the force location is not valid anymore. Liu and Ewins [40] proved it mathematically considering two subsystems linked by two springs on different DOFs as illustrated on Figure 21.

These parallel links represent the fact that more than one vibration path is available. Physically it can represent the coupling between rotational and transverse motion in Euler-Bernoulli beams. In this work the authors show that if the response of system A is measured at two DOFs, $j_{1}$ and $j_{2}$, the ratio of two FRFs, $H_{j 1 k} / H_{j 2 k}$ is dependent on the input location $k$. This dependence on force location restricts the use of TFs for damage detection and localization to cases in which the input locations do not change. In addition, equation (15) is based on the tridiagonal nature of matrices $\mathbf{K}$ and $\mathbf{M}$. For dispersive systems, the matrices are not tridiagonal and such a result does not hold anymore, so that even in the ideal case (damage next to the excitation location), localization cannot be proved. In fact, the zeros will be sensitive to all the parameters of the structure, and not only to a subset of parameters, as it was the case for nondispersive systems.

\subsection{Damage localization: Illustrative example}

In order to illustrate this, an Euler Bernoulli beam is modeled using the finite element method. This class of systems is 
governed by a fourth order differential equation and the discrete representation results in matrices which are not tri-diagonal. This means that nodes have more than one connection to others nodes or that that the connections are not only to their nearest neighbors (as illustrated Figure 21).

We consider a cantilever beam of length $\mathrm{L}=1.12 \mathrm{~m}$, rectangular cross section of width $1.18 \mathrm{~cm}$ and height $0.635 \mathrm{~cm}$. The beam is made of fiberglass which has a density $1620 \mathrm{~kg} / \mathrm{m} 3$ and a Young's modulus $\mathrm{E}=22.4 \mathrm{GPa}$ and is identical to the one used in [25]. It is discretized with 16 EulerBernoulli finite elements as illustrated Figure 22. A transverse force is applied on node 13, element 11 is damaged with a bending stiffness reduction of $5 \%$, and 8 transverse displacements are used as outputs for $\mathrm{TF}$ computation.

This case corresponds to the ideal one for damage localization as illustrated in Figure 11 for mass-spring systems. The frequency band includes the 8 first flexural modes of the beam. Figure 23 shows the evolution of the 7 damage indicators (computed with (16)) as a function of $\omega$. Damage contained between $\mathrm{x}_{5}$ and $\mathrm{x}_{6}$ should appear in $\mathrm{D}_{56}$. Clearly, although damage is detected, it cannot be localized, except in a very small frequency range around $150 \mathrm{rad} . \mathrm{s}^{-}$ ${ }^{1}$ between the second and the third mode of the structure.

\section{Conclusions}

The first part of this paper was devoted to a critical review on the use of TFs for damage detection and localization. The review highlights the importance of the choice of the frequency bands, the effects of environment, and the dependency on the force location. It also highlights the lack of analytical results to determine the practical limitations when using TFs for damage detection and localization.
In the second part of the paper, we have studied non dispersive systems such as chainlike mass-spring systems and derived an analytical formula linking the $\mathrm{TF}$ to the properties of four subsystems, defined by the boundary conditions, the excitation location, and the position of the two points used for the computation of the TF. This formula shows that damage localization is only guaranteed when the excitation force is located exactly next to the damage, which is very rarely the case in practice. In all other cases, although damage localization is not guaranteed, it seems to work when restricting the frequency band to frequencies lower than the first eigenfrequency of the system. The study also shows that locally changing the mass of the system has not the same impact as changing the stiffness, especially at low frequencies, so that replacing damage by an added mass is not advised when using TFs.

The analytical results derived for nondispersive systems cannot be extended to more general dispersive systems such as beams or plates. For such systems, damage localization is therefore not guaranteed, even for the ideal case in which the excitation is located next to the damage. This is illustrated on a simple beam example in the last section of this paper.

A general conclusion to this study is that extreme care should be taken when using TFs for damage detection and localization. While it is obvious that such functions are sensitive to damage, it seems to be difficult to use them in an unsupervised manner, i.e. without knowing a priori how they will be affected by damage, environment, and the excitation location. 


\section{References}

[1] A. Rytter, Vibration based inspection of civil engineering structures, $\mathrm{Ph}$. D. Thesis, Departement of Building and structural Engineering, Aalborg University, Denmark, (1993).

[2] S.W. Doebling, C.R. Farrar, M.B. Prime and D.W. Shevitz, Damage identification and health monitoring of structural and mechanical systems from changes in their vibration characteristics: a literature review, Los Alamos Natinal Laboratory Report, A-13070-VA5 (1996)

[3] B. Peeters, J. Maeck and G. De Roeck Vibration-based damage detection in civil engineering: excitation sources and temperature effects. Smart Materials and Structures, 10(3):518-527 (2001).

[4] K. Worden, G. Manson and N.R.J. Fieller, Damage detection using outlier analysis, Journal of Sound and vibration 229(3), 647-667(2000)

[5] J. Kullaa, Damage detection of the Z24 bridge using control charts, Mechanical Systems and Signal Processing, 17(1), 163170 (2003).

[6] A. Deraemaeker, E. Reynders, G. De Roeck, and J. Kulla, Vibration based structural health monitoring using outputonly measurements under changing environment, Mechanical Systems and Signal Processing 22, 34-56, (2008).

[7] A. Teughels, G. De Roeck, Structural damage identification of the highway bridge Z24 by FE model updating. Journal of Sound and Vibration, Volume 278, Issue 3, 589610, (2004).

[8] A.K. Pandey, M. Biswas, and M.M. Samman. Damage detection from changes in curvature mode shapes. Journal of Sound and Vibration, 145(2):321-332, 1991.

[9] P. Cornwell, S.W. Doebling, and C.R. Farrar. Application of the strain energy damage detection method to plate-like structures. Journal of Sound and Vibration, 224(2):359-374, 1999
[10] J. Maeck and G. De Roeck. Dynamic bending and torsion stiffness derivation from modal curvatures and torsion rates. Journal of Sound and Vibration, 225(1):153-170, 1999.

[11] A. Deraemaeker. On the use of dynamic strains and curvatures for vibration based damage localization. In Proc EWSHM 2010, Sorento, Italy, July 2010

[12] Q. Chen, Y.W Chan, K Worden and G.R. Tomlinson, Structural fault detection using neural networks trained on transmissibility functions. In Proceedings of the International Conference on Vibration Engineering, Beijing, China, pp 456-646 (1994)

[13] K. Worden Structural Fault Detection Using a Novelty Measure. Journal of Sound and Vibration.1997;201(1):85-101.

[14] K Worden, G Manson, N.R.J. Fieller, Damage Detection Using Outlier Analysis. Journal of Sound and Vibration. 2000;229(3):647-667.

[15] K. Worden Experimental Validation of a Structural Health Monitoring Methodology: Part I. Novelty Detection on a Laboratory Structure. Journal of Sound and Vibration. 2003;259(2):323-343.

[16] G. Manson Experimental Validation of a Structural Health Monitoring Methodology: Part Ii. Novelty Detection on a Gnat Aircraft. Journal of Sound and Vibration. 2003;259(2):345-363.

[17] Q. Chen, Y.W. Chan. and K. Worden Structural fault diagnosis and isolation using neural networks based on response-only data. Computers \& Structures. 2003;81(2223):2165-2172.

[18] G. Manson. Experimental Validation of a Structural Health Monitoring Methodology: Part Iii. Damage Location on an Aircraft Wing. Journal of Sound and Vibration. 2003;259(2):365-385.

[19] C. Devriendt, G. Steenhackers and P. Guillaume, On the use of Transmissibility in the field of: operational Modal Analysis and Structural Health Monitoring Proceedings of the $8^{\text {th }}$ National Congress in theoretical and applied Mechanics, May 28-29 Brussels, Belgium (2009). 
[20] C. Devriendt, P. Guillaume. The use of transmissibility measurements in output-only modal analysis. Mechanical Systems and Signal Processing. 2007.

[21] K. Worden, L.Y. Cheung., J.A Rongong. Damage detection in an aircraft compoment model. In Proc International modal analysis conference $N^{\circ} 19$, Kissimmee Florida, USA, pp 1234-1241 (2001).

[22] E. Papatheou, G. Manson, RJ. Barthorpe, K. Worden. The use of pseudofaults for novelty detection in SHM. Journal of Sound and Vibration. 2010;329(12):23492366.

[23] MJ. Schulz, AS. Abdelnaser, PF. Pai, MS. Linville, J. Chung. Detecting structural damage using transmittance functions. International Modal Analysis Conference, Orlando, Florida. pp 638-644 (1997).

[24] MJ. Schulz, PF. Pai, DJ. Inman. Health monitoring and active control of composite structures using piezoceramic patches. Composites: Part B 1999;30:713-25.

[25] H. Zhang, M. J. Schulz, F. Ferguson and P. F. Pai. Structural Health Monitoring Using Transmittance Functions. Mechanical Systems and Signal Processing. 1999;13(5):765-787.

[26] A. Ghoshal, MJ. Sundaresan, MJ. Schulz, PF. Pai. Structural Health Monitoring Techniques for wind turbine blades. Journal of Wind Engineering and Industrial Aerodynamics. 2000;85:309-324.

[27] V.Caccese Detection of bolt load loss in hybrid composite/metal bolted connections. Engineering Structures. 2004;26(7):895-906.

[28] W. Liu and D J Ewins, Transmissibility Properties of MDOF Systems, In Proc of International modal analysis conference $N^{o} 16$, Santa Barbara CA , USA, pp. 847-854 (1998).

[29] S. Ribeiro a. on the Generalisation of the Transmissibility Concept. Mechanical Systems and Signal Processing. 2000;14(1):29-35.

[30] NMM. Maia. the Transmissibility Concept in Multi-Degree-of-Freedom
Systems. Mechanical Systems and Signal Processing. 2001;15(1):129-137.

[31] J Mottershead. on the Zeros of Structural Frequency Response Functions and Their Sensitivities. Mechanical Systems and Signal Processing. 1998;12(5):591-597.

[32] T. J. Johnson and D. E. Adams, Transmissibility as a differential indicator of structural damage, Journal of Vibration and Acoutic, transactions of the ASME, Vol124, Octobre2002, 634-641 (2002).

[33] RPC. Sampaio, NMM. Maia, S.Ribeiro Transmissibility techniques for damage detection. In Proc International modal analysis conference $N^{\circ} 19$, Kissimmee Florida , USA, pp. 1524-1527 (2001).

[34] NMM. Maia, R. Almeida, APV. Urgueira, RPC. Sampaio. Damage detection and quantification using transmissibility. Mechanical Systems and Signal Processing. 2011;25(7):2475-2483.

[35] T. J. Johnson, R. L. Brown, D. E. Adams and Mark Schiefer, Distributed structural health monitoring with a smart sensor array, Mechanical Systems and Signal Processing 18 (2004) 555-572.

[36] T.J. Johnson, D.E. Adams, Rolling tire diagnostic experiments for identifying incipient bead damage using time, frequency, and phase plane analysis, in: Proceedings of the SAE 2006 World Congress, 2006, SAE Paper Number 2006-01-1621.

[37] H. Kess,D. Adams; Investigation of operational and environmental variability effects on damage detection algorithms in a woven composite plate. Mechanical Systems and Signal Processing. 2007;21(6):23942405

[38] ZQ. Lang, G. Park, CR. Farrar, et al. Transmissibility of non-linear output frequency response functions with application in detection and location of damage in MDOF structural systems. International Journal of Non-Linear Mechanics. 2011;46(6):841-853.

[39] D. K. Miu, Mechatronics Electromechanics and controlmechanics, Springer-Verlag, 1993. 
[40] W. Liu and D J Ewins, Transmissibility Properties of MDOF Systems, In Proc of International modal analysis conference $N^{o} 16$, Santa Barbara CA , USA, pp. 847-854 (1998). 


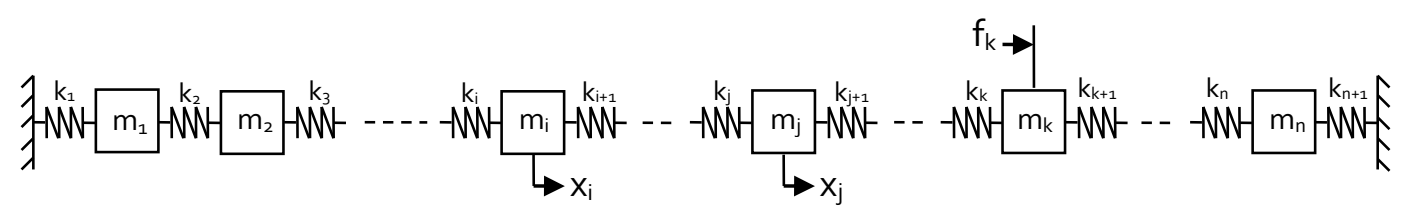

Figure 1: A n DOF mass spring system

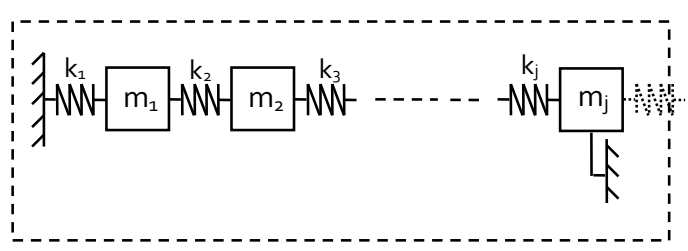

$\mathrm{Ma}_{\mathrm{j}}$

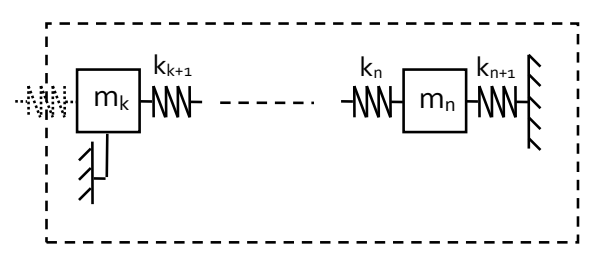

$M c_{k}$

Figure 2:Subsystems of mass spring system zeros

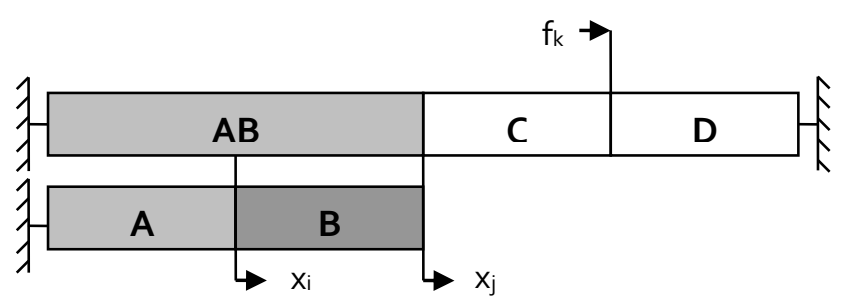

Figure 3: Mass spring system, subsystem representation 

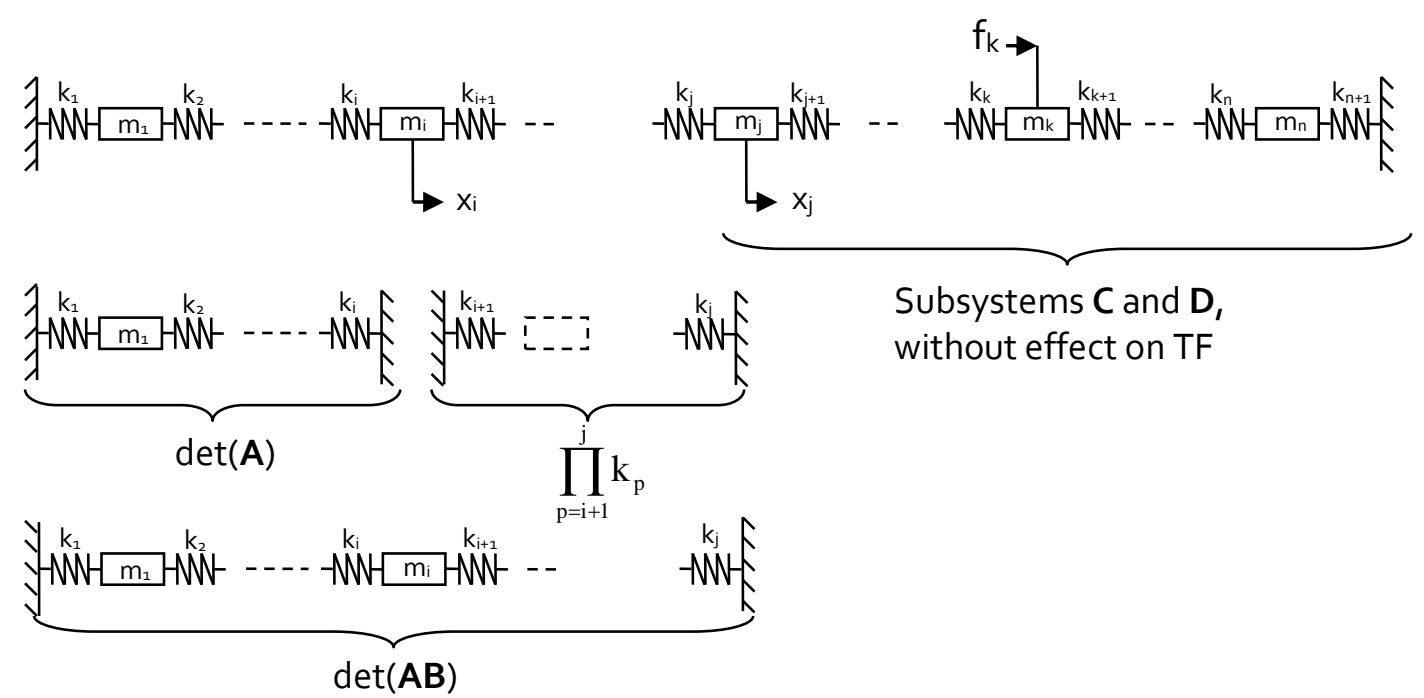

Figure 4: Subsystems involved in TF computation

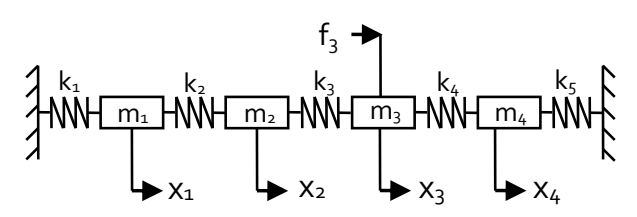

Figure 5 : A 4 DOF mass spring system
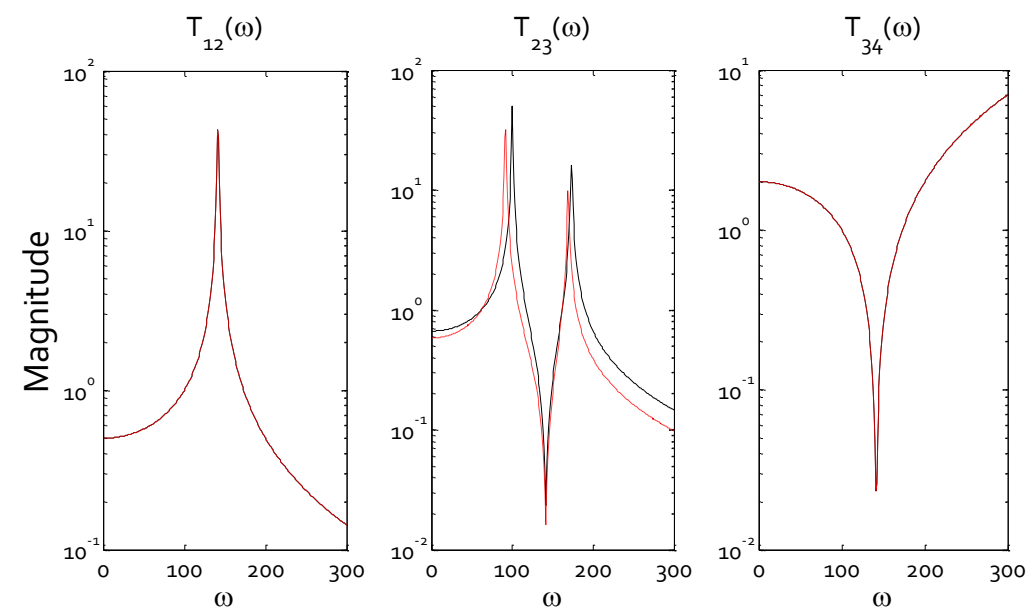

Figure 6: Transmissibility functions, $k_{3}$ is damaged (Black line: Healthy structure, Red dotted line: damaged structure) 


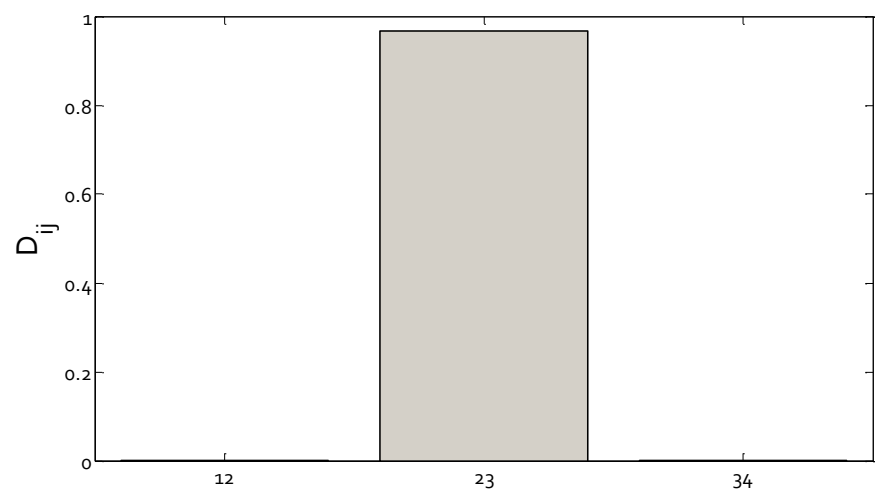

Figure 7: Damage indicator: $k_{3}$ is damaged

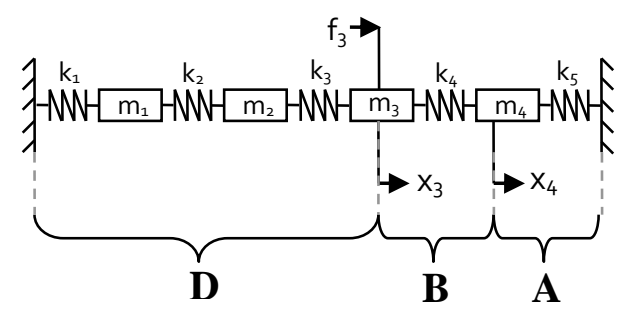

Figure 8: Subsystem representation for $T^{3} 34$
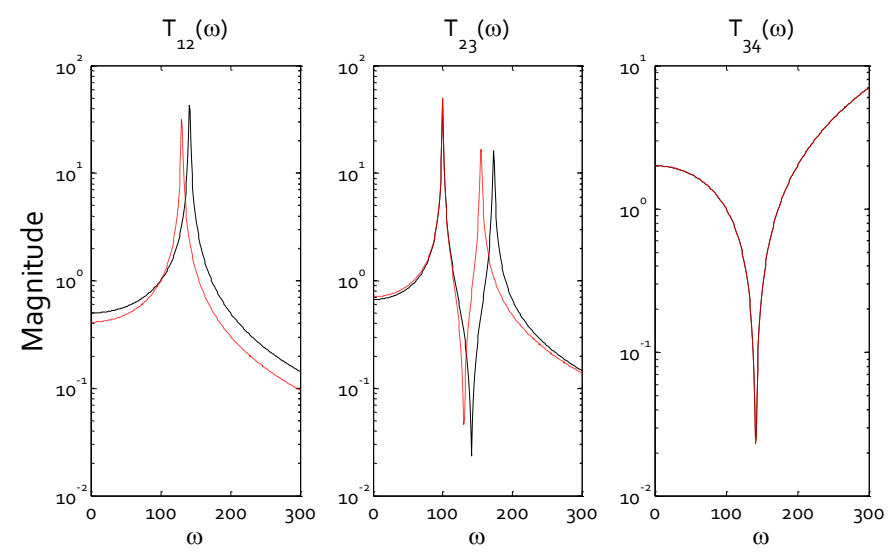

Figure 9:Transmissibility functions, $k_{2}$ is damaged (Black line: Healthy structure, Red dotted line: damaged structure) 


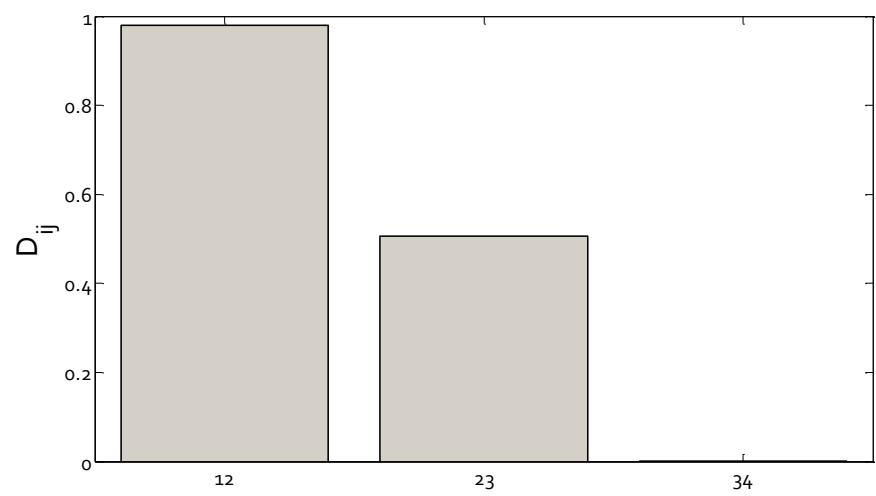

Figure 10: Damage indicator: $k_{2}$ is damaged

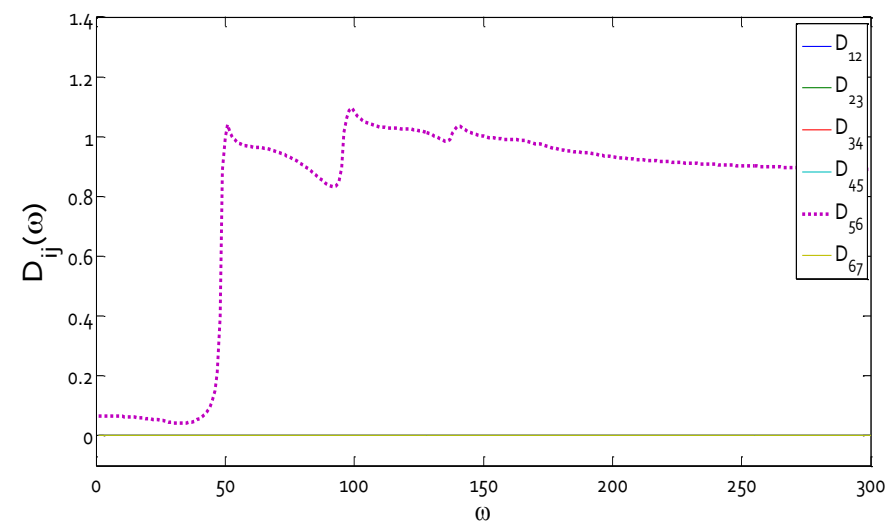

Figure 11: Evolution of the six damage indicators as a function of $\omega$ for a stiffness reduction of $30 \%$ on $\mathrm{k}_{6}$

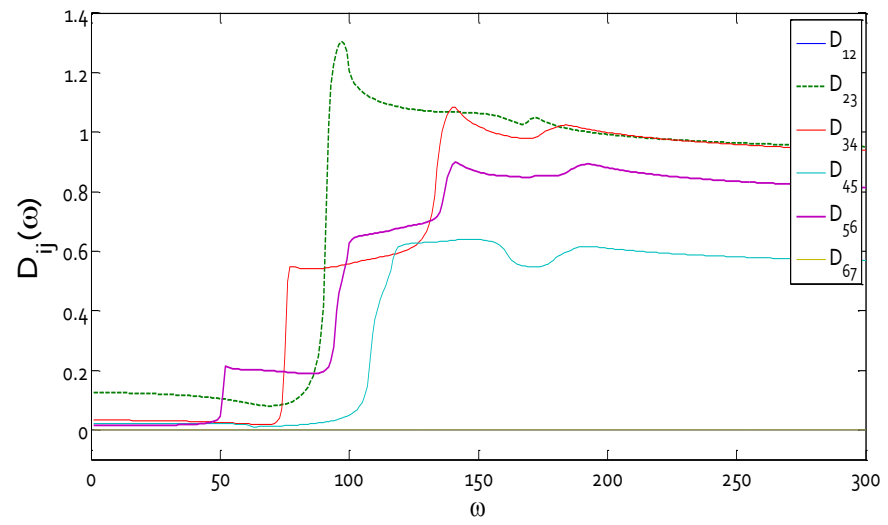

Figure 12: Evolution of the six damage indicators as a function of $\omega$ for a stiffness reduction of $30 \%$ on $k_{3}$ 


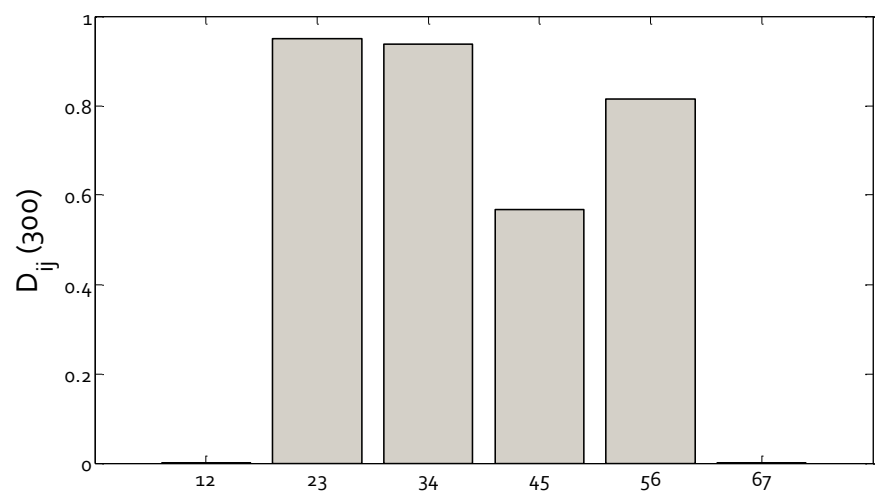

Figure 13: Damage indicator: $k_{3}$ is damaged. $\omega \in[0,300]$

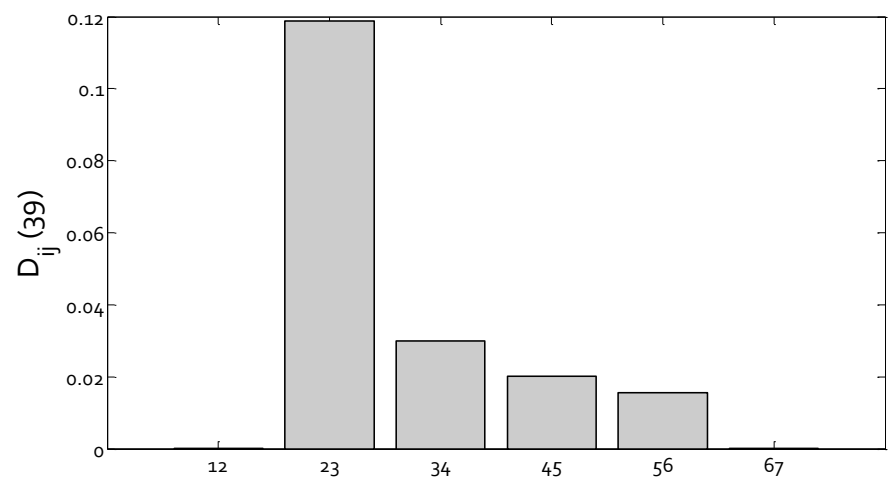

Figure 14: Damage indicator: $k_{3}$ is damaged $\omega \in[0,39]$

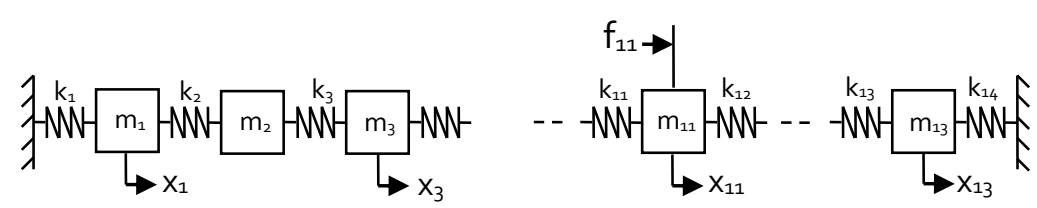

Figure 15: A 13 DOF mass spring system 


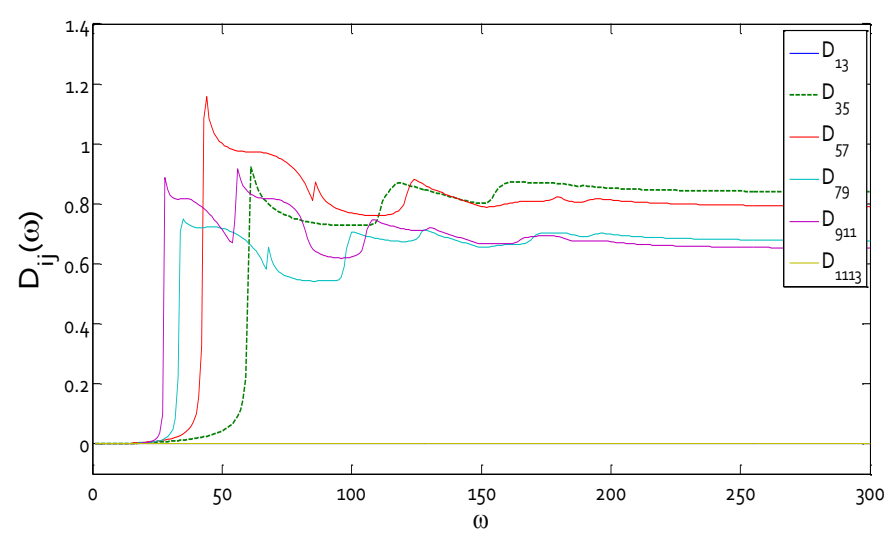

Figure 16: Evolution of the six damage indicators as a function of $\omega$ for a mass add of $30 \%$ on $\mathrm{m}_{4}$

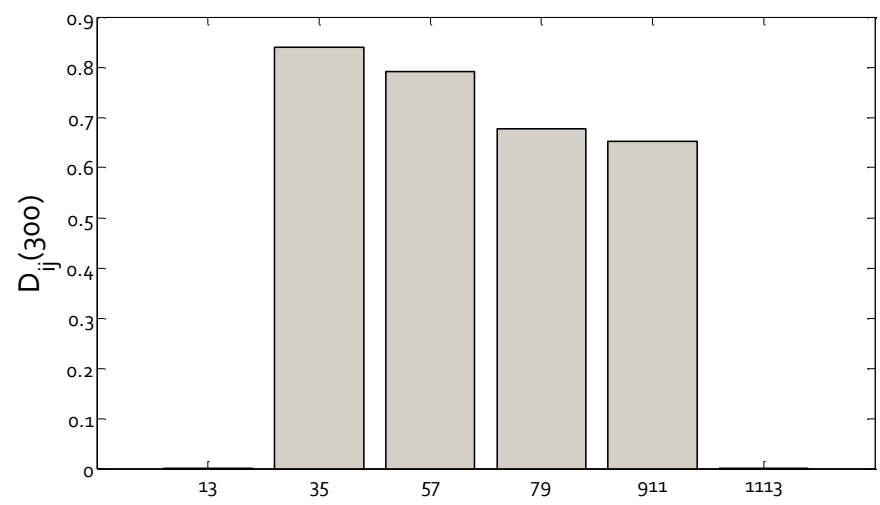

Figure 17: Damage indicator: $\boldsymbol{m}_{4}$ is increased $\omega \in[0,300]$

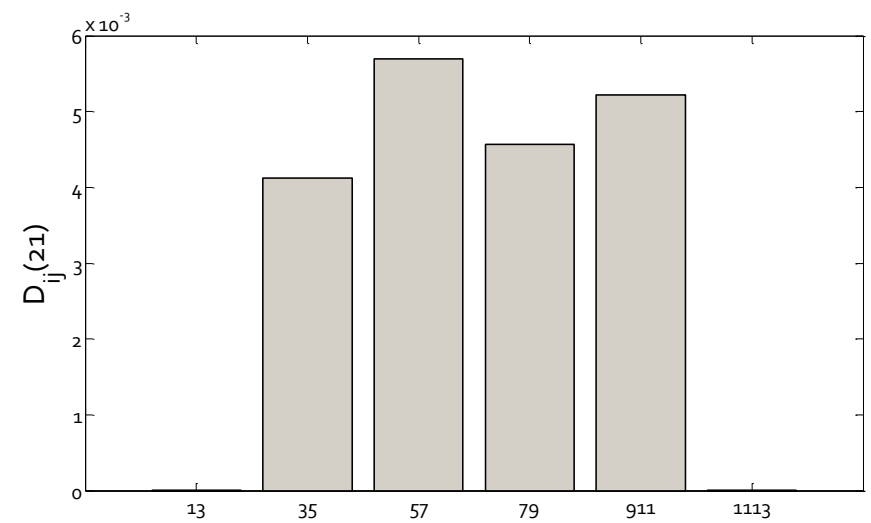

Figure 18: Damage indicator: $m_{4}$ is increased $\omega \in[0,21]$ 


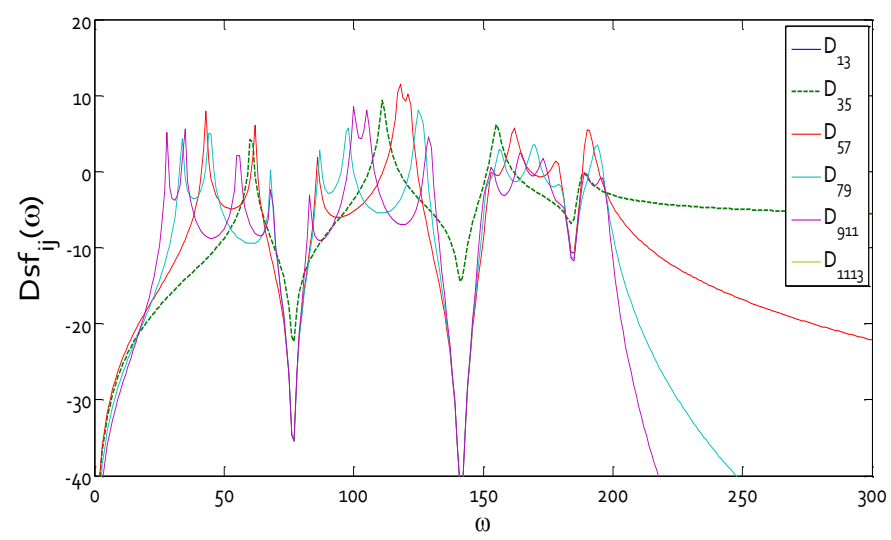

Figure 19: "Single frequency" Damage indicator $(d B): m_{4}$ is increased

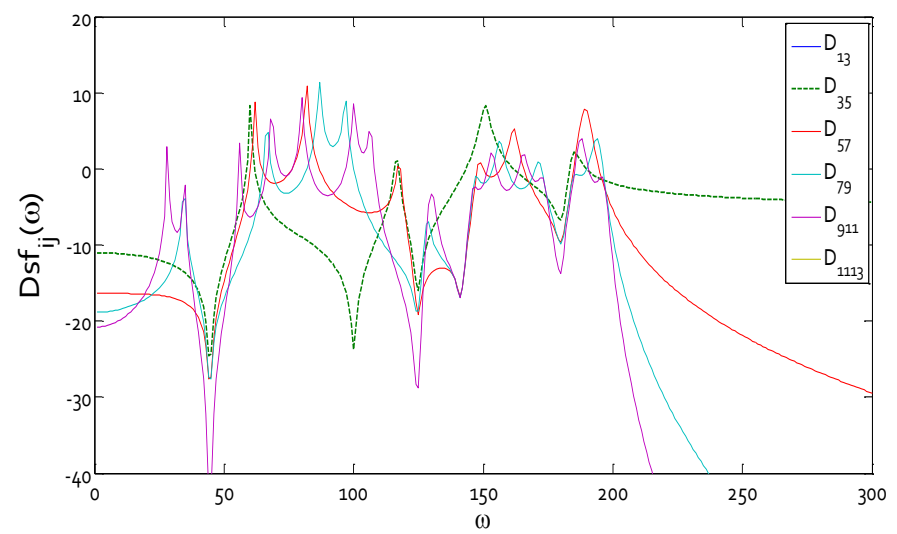

Figure 20: "Single frequency" Damage indicator $(d B): k_{4}$ is damaged

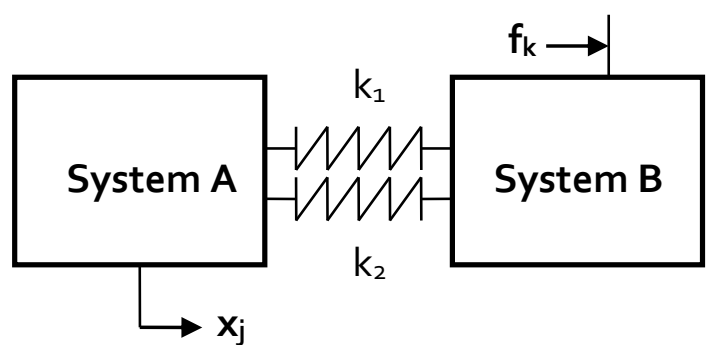

Figure 21: Two systems connected with two springs: "=" connection 


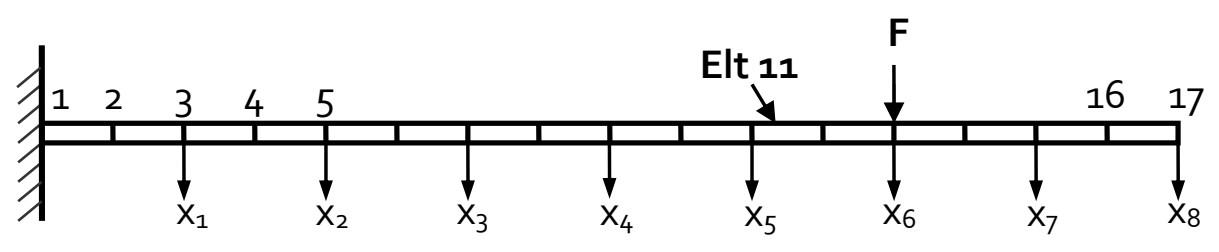

Figure 22: Cantilever beam discretized with 16 finite elements, element 11 is damaged

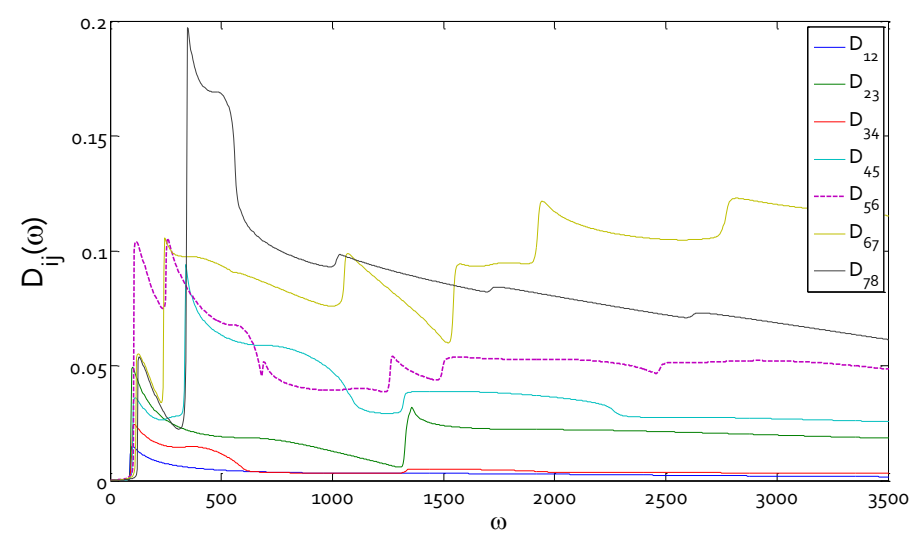

Figure 23: Evolution of the seven damage indicators as a function of $\omega$ for a bending stiffness reduction of $5 \%$ on element 11 